\title{
Predictors of VT recurrence in patients with VT inducibility at the end of radiofrequency ablation: Should we use VT non-inducibility as a routine endpoint?
}

\author{
Kazutaka Nakasone $^{1}$, Koji Fukuzawa ${ }^{1}$, Kunihiko Kiuchi ${ }^{1}$, Mitsuru Takami ${ }^{1}$, jun sakai ${ }^{1}$, \\ Toshihiro Nakamura ${ }^{2}$, atsusuke yatomi ${ }^{1}$, Yusuke Sonoda ${ }^{1}$, Hiroyuki Takahara ${ }^{1}$, Kyoko \\ Yamamoto $^{1}$, Yuya Suzuki ${ }^{3}$, Kenichi Tani ${ }^{4}$, Hidehiro Iwai ${ }^{1}$, Yusuke Nakanishi ${ }^{1}$, and Ken-ichi \\ Hirata $^{1}$ \\ ${ }^{1}$ Kobe University Graduate School of Medicine \\ ${ }^{2}$ Kobe University \\ ${ }^{3}$ Akashi Medical Center \\ ${ }^{4}$ Kobe University Hospital
}

December 6, 2021

\begin{abstract}
Introduction: It has been reported that ventricular tachycardia (VT) non-inducibility at the end of ablation is associated with less likely VT recurrence. However, it is not clear whether we should use VT non-inducibility as routine end point in VT ablation. The aim of this study was to evaluate VT recurrence in patients in whom VT non-inducibility could not be achieved at the end of the RF ablation and the factors attributing to the VT recurrence. METHODS and RESULTS: We analyzed 84 consecutive patients that underwent RF ablation, and 64 patients in whom VT non-inducibility could not be achieved were studied. The primary endpoint was recurrence of any sustained VT during the follow-up. During a median follow-up period of 1.4 years (IQR:0.3-2.0), 22 (34\%) of the cases had VT recurrences. In the multivariate analysis showed that an LVEF[?]35\% (HR:0.21; 95\% CI:0.07- 0.54; P<0.01) and successful identification and ablation of all clinical VT isthmuses (HR:0.21; 95\% CI:0.03- 0.72; $\mathrm{P}=0.01$ ) were independent predictors of fewer VT recurrences. RF ablation was associated with a $91.1 \%$ reduction in VT episodes. CONCLUSION: Even if VT non-inducibility could not be achieved, the patients with LVEF[?]35\% or in whom all clinical VT isthmuses could successfully be identify and ablated might be prevented from having VT recurrences. The validity of VT non-inducibility of any VT should be evaluated by each patient's background and the results of the procedure.
\end{abstract}

Predictors of VT recurrence in patients with VT inducibility at the end of radiofrequency ablation: Should we use VT non-inducibility as a routine endpoint?

Kazutaka Nakasone $^{1}$, MD, Koji Fukuzawa ${ }^{*}{ }^{1,2}$, MD, PhD, Kunihiko Kiuchi ${ }^{1}{ }^{1}{ }^{2}$, MD, PhD, Mitsuru Takami ${ }^{1}$, MD, PhD, Jun Sakai ${ }^{1}$, MD, Toshihiro Nakamura ${ }^{1}$, MD, Atsusuke Yatomi ${ }^{1}$, MD, Yusuke Sonoda ${ }^{1}$, MD, Hiroyuki Takahara ${ }^{1}$, MD, Kyoko Yamamoto ${ }^{1}$, MD, Yuya Suzuki ${ }^{1}$, MD, Kenichi Tani ${ }^{1}$, MD, Hidehiro Iwai ${ }^{1}$, MD, Yusuke Nakanishi ${ }^{1}$, MD, Ken-ichi Hirata ${ }^{1}, \mathrm{MD}, \mathrm{PhD}$

1 Division of Cardiovascular Medicine, Department of Internal Medicine, Kobe University Graduate School of Medicine, Kobe, Japan

2 Section of Arrhythmia, Division of Cardiovascular Medicine, Department of Internal Medicine, Kobe University Graduate School of Medicine, Kobe, Japan

*Corresponding Author 


\section{Author contributions:}

Kazutaka Nakasone: ,,,.,,,clinical practice/data sampling/drafting article

Koji Fukuzawa: ,,,, ,., , concept/design/data analysis/interpretation/drafting article

Kunihiko Kiuchi: ,,,,,,data collection/statics

Mitsuru Takami: ,,,,,,data collection/statics

Jun Sakai: ,,,,,,data collection/statics

Toshihiro Nakamura: ,,,.,,data collection/statics

Atsusuke Yatomi: ,,,,, data collection/statics

Yusuke Sonoda: ,,,,., data collection/statics

Hiroyuki Takahara: ,,,,,,data collection/statics

Kyoko Yamamoto: ,,,,,,data collection/statics

Yuya Suzuki: ,,,,,,data collection/statics

Kenichi Tani: ,,.,., data collection/statics

Hidehiro Iwai: ,,,,,,data collection/statics

Yusuke Nakanishi: ,,,.,,data collection/statics

Ken-ichi Hirata: approval of article

Conflict of Interest : The Section of Arrhythmia is supported by an endowment from Abbott JAPAN and Medtronic JAPAN and has received a scholarship fund from Biotronik JAPAN. Ken-ichi Hirata chairs the Section, and Koji Fukuzawa and Kunihiko Kiuchi belong to the Section. However, all authors report no conflict of interest for this manuscript's contents.

Sources of Funding: There are no sources of funding related to this study

Total word count: 4062 words

Corresponding Author: Koji Fukuzawa, MD, PhD

Affiliation: Section of Arrhythmia, Division of Cardiovascular Medicine, Department of Internal Medicine, Kobe University Graduate School of Medicine

Address: 7-5-2 Kusunoki-Cho, Chuoh-Ku, Kobe 650-0017, Japan

Telephone: +81-78-382-5846, Fax: +81-78-382-5859

E-mail: kfuku@med.kobe-u.ac.jp

Data availability statement: The data that support the findings of this study are available from the corresponding author upon reasonable request.

\section{Introduction}

Radiofrequency (RF) ablation is a well-established treatment for recurrent ventricular tachycardia (VT) and many studies have shown that a successful RF ablation can prevent VT recurrence and reduce mortality in patients with structural heart disease (SHD). ${ }^{1-3}$

In the early 1990s, the success of RF ablation was based on the confirmation of clinical VT non-inducibility. ${ }^{4}$ After that, in the era of 3D mapping system, various indicators such as the disappearance of abnormal potentials was proposed, ${ }^{5}$ but VT non-inducibility is still one of the most important endpoints. ${ }^{6-9}$ Previous studies have demonstrated that VT non-inducibility at the end of RF procedures is less likely to be associated 
with a VT recurrence in patients with ischemic cardiomyopathy (ICM) ${ }^{6}$ or non-ICM (NICM). ${ }^{7}$ However, it is not clear whether we should use VT non-inducibility as a routine endpoint of VT ablation. In sicker patients, the risk of repeated induction tests, a prolonged operative time, and overtreatment should be considered. In clinical practice, it may not be realistic to achieve non-inducibility in some patients.

The primary purpose of this study was to evaluate VT recurrence in patients in whom VT non-inducibility could not be achieved at the end of the RF ablation and the factors attributed to VT recurrences in ICM and NICM patients. Further, the impact of the clinical VT ablation on the outcomes was also assessed as the secondary purpose.

\section{Methods}

\subsection{Study Population}

From January 2009 to April 2020, 84 consecutive patients with SHD underwent RF ablation (116 procedures) for drug-resistant VT in our hospital. Patients who underwent multiple procedures were evaluated during the last procedure.

VT non-inducibility was achieved in 20 patients but it was not achieved in 64 . To evaluate the validity of "VT non-inducibility" as an endpoint of RF ablation, 64 patients in whom VT non-inducibility was not achieved were studied. The study was approved by the ethics committee of Kobe University Hospital, and all patients gave their written informed consent.

\subsection{Ablation Procedure}

The procedures were mostly performed under conscious sedation, but it was done under general anesthesia in 7 cases. Transvenous multipolar catheters were placed into the cardiac chambers appropriate for the arrhythmia being studied (right and/or left ventricular [RV and/or LV]). LV mapping was performed via the retrograde aortic or transseptal approach. When necessary, an epicardial approach using a percutaneous subxiphoid puncture was attempted at the beginning of the procedure as previously described. ${ }^{10}$ Electroanatomic mapping systems such as CARTO3 (Biosense Webster, Diamond Bar, CA, USA) or Ensite (Abbott, ST. Paul, MN, USA) were used. Intracardiac echocardiography assisted in defining the anatomical structures, monitoring for potential complications, and performing transseptal punctures. Systemic anticoagulation was achieved with intravenous heparin targeted to a minimum activation clotting time of 350 seconds during LV and 250 seconds during RV mapping. The radiofrequency current was delivered with a 3.5-mm open irrigated tip catheter, with power settings of 30 to $50 \mathrm{~W}$ and a temperature limit of 43 . Contact force sensing catheters were used by the operators while aiming for a $5-30 \mathrm{~g}$ contact force.

\subsection{Mapping, Ablation strategy, and the induction protocol}

Firstly, substrate mapping was performed during sinus rhythm (SR) or ventricular pacing. The areas of abnormal electrograms, such as fractionated or late potentials, were tagged to denote the type of electrogram, and pace mapping was performed in those areas. Pace mapping was also performed at the presumed isthmus and exit regions of the clinical VT. The QRS morphology and stimulus to QRS (St-QRS) interval were evaluated. At the area of interest, a pace map was performed at maximum output $(1.0 \mathrm{~ms}, 20 \mathrm{~V})$, and the output was decreased until pacing could no longer capture the myocardium to evaluate the functional pace map response.

Secondly, programmed ventricular stimulation was delivered with up to double extra-stimuli at two different basic cycle lengths (CLs) (600 and 400 milliseconds) from at least two sites (the right ventricular apex, outflow tract, or left ventricle). If the induced VT was hemodynamically tolerated, activation mapping was acquired, and entrainment pacing was delivered if possible. In cases of hemodynamically unstable VT, the tachycardias were interrupted by overdrive pacing or direct current (DC) defibrillator. When VT was not induced, the induced VT was hemodynamically unstable, or the VT was not sustained, pace mapping was performed and compared to the clinical VT morphology to confirm the VT exit. 
The VT isthmus was defined as sites where mid-diastolic potentials (MDPs) during the VT were present, and the RF ablation terminated the VT or pace mapping showed multiple exit sites (MESs) ${ }^{11} \mathrm{RF}$ ablation was applied at the critical isthmus of the target VT during VT, based on the findings from activation, entrainment, and pace mapping. For hemodynamically unstable VTs, RF ablation was applied at sites with abnormal electrograms, a longer St-QRS, and the presence of a functional pace map response. Fundamentally, all the clinical VTs were targeted in all cases. The non-clinical VTs were also targeted in cases with electrical storms.

Finally, programmed ventricular stimulation was delivered with up to double extra-stimuli at two different basic CLs from at least two sites, down to $200 \mathrm{~ms}$ or until the refractory period.

\subsection{The definition of non-inducibility and the impact of the clinical VT}

At the end of the RF ablation, in those that no sustained VT could be induced, they were designated with VT non-inducibility. The patients in whom only non-sustained VT (termination within 30 seconds) or ventricular fibrillation (VF) was induced, were also classified as having VT non-inducibility. In contrast, if sustained VT was induced, regardless of whether it was clinical or non-clinical, the patient was judged as having residual VT inducibility. Among the patients in whom VTs were sustained or induced at baseline, the patients that did not undergo a VT induction test or only received an inadequate VT induction test at the end of the procedure due to the patient's condition, were also classified as having residual VT inducibility. The induced VTs were defined as being clinical if they matched the arrhythmia captured clinically on the 12 lead ECG or, in cases where no ECG was available, they matched the tachycardia cycle length of the implantable cardioverter defibrillator log (difference $<30 \mathrm{~ms}$ ).

In order to analyze the impact of the clinical VT inducibility at the end of the procedure, the patients were classified into 2 groups according to the presence or absence of inducibility of the clinical VT at the end of the procedure: Group A - all clinical VT isthmuses were identified and ablated, and those VTs could no longer be induced thereafter (Figure 2), and Group B - none or some of the clinical VT isthmuses could not be identified or were identified and ablated but remained inducible.

\subsection{Follow-up}

Clinical follow-up visits and implantable cardioverter defibrillator (ICD) interrogations were scheduled at 1 , 3,6 , and 12 months after the procedure, with remote device monitoring when possible.

\subsection{VT burden}

The pre-procedure VT burden was defined as the number of VT episodes and shock therapies up to 2 years from the time of the ablation procedure. The post-procedure VT burden was determined after 2 years from the day of ablation procedure. In redo procedures, the pre-procedure VT burden was defined as the number of VT episodes occurring between the previous ablation procedure and the when the redo ablation procedure was performed. VT episodes were defined as continuous VT for 30 seconds and/or a syncopal events, or as VT that required an appropriate intervention for termination. We also defined incessant VT that persisted or immediately reoccurred despite appropriate intervention to terminate the VT.

\subsection{Statistical Analysis}

Continuous variables are presented as either means $( \pm \mathrm{SD})$ or medians (with interquartile ranges $[\mathrm{IQR}])$ and categorical variables as numbers and percentages (\%). Differences in the continuous variables between the 2 groups were assessed by an unpaired t test or Mann-Whitney test. Categorical variables were compared through a Fisher exact test. The estimated event free survival probabilities were calculated using a KaplanMeier analysis; log-rank statistics were used for group comparisons. The follow-up period from the last procedure was calculated with the median value and interquartile range. At first, to assess the clinical predictors of VT-free survival and survival from cardiac death, a univariable Cox proportional analysis was performed. Sequentially, variables with a $\mathrm{P}<0.10$ in the univariable analysis were included in the multivariable 
analysis, and the hazard ratios (HR) and $95 \%$ CI were calculated. A P $<0.05$ was considered statistically significant. All statistical analyses were performed using SAS 9.4 software (SAS Institute, Cary, NC).

\section{Result}

\subsection{Patient Characteristics}

During the study period, 84 patients underwent VT ablation, and 64 in whom VT non-inducibility was not achieved at the end of the procedure were studied (Figure 1). The baseline patient characteristics are summarized in Table 1 . The mean age was $66 \pm 14$ years, and 54 patients $(84 \%)$ were male. The average LV ejection fraction (LVEF) was $38 \pm 13 \%$.

The most frequent underling heart disease of the VT was ischemic cardiomyopathy (ICM; 38\%); sarcoidosis $(23 \%)$ and dilated cardiomyopathy (19\%) were also common. Thirty-one patients (48\%) had electrical storms before the RF ablation, and 19 (30\%) had persistent incessant VT at the beginning of the RF procedure.

\subsection{Procedural Details}

Table 2 reports the procedural data of the VT ablation performed in this study. An epicardial access was required in $13(20 \%)$ patients. Forty-one patients (64\%) underwent a first procedure, $18(28 \%)$ a second, and $2(3 \%)$ a third. The mean procedural and RF times were $246 \pm 66$ and $31 \pm 20$ minutes, respectively. The electroanatomical maps were created with CARTO in $86 \%$ of the cases and Ensite in $13 \%$. The 3D mapping system was not used in 1 case $(2 \%)$ because of the influence from a ventricular assist device.

\subsection{Procedural Outcomes}

The number of VT morphologies documented prior to ablation, which were considered clinical VTs, was 1 (IQR, $1-1$; range, $1-5$; total 83) (Table 1), and the number of VT morphologies documented during the procedure was 2 (IQR, 1 - 2; range, 0 - 10; total 126) (clinical VT was 1 [IQR, 1 - 1; range, 0 - 5; total 66], and non-clinical VT was 1 [IQR, $0-1$; range, $0-9$; total 60].) (Table 2).

Among the 83 clinical VTs, 19 (23\%) persisted incessantly at the beginning of the ablation, 47 (57\%) were induced during the procedure, and $17(20 \%)$ were not induced during the procedure (Supplementary Table 1.). Among the 66 clinical VTs, which were documented during the procedure, an adequate activation map could be acquired in 24 VTs $(36 \%)$. The critical isthmus could be identified in 35 clinical VTs (53\%); for 26 VTs non-inducibility after ablation could be achieved, and 9 VTs could not be eliminated because the isthmus was deep (7 VTs) or a coronary artery was nearby (2 VTs).

Table 3 shows the ablation data per patient. All clinical VT isthmuses were identified and ablated, and those VTs could not be induced thereafter in 18 patients (28\%, Group A); 12 patients (19\%) were found by the presence of MDPs and VT termination during the RF ablation, and 6 patients (9\%) were found by MESs. On the other hand, all or some clinical VT isthmuses could not be identified or were identified and ablated but remained inducibility in 46 patients (72\%, Group B). Among them, 8 patients (13\%) underwent ablation after identifying the clinical VT isthmus, but the ablation failed because the isthmus was deep (6 patients) or a coronary artery was nearby (2 patients). In 4 patients $(6 \%)$, some but not all clinical VT isthmuses were ablated, and in 2 patients (3\%) only a nonclinical VT isthmus ablation was performed. Further, thirty patients $(47 \%)$ underwent only a substrate ablation because no VT isthmuses could be identified.

\subsection{Procedural complications}

Cardiac tamponade occurred in 1 case $(2 \%)$ and required drainage. One groin hematoma $(2 \%)$ was conservatively managed (Table 2 ).

\subsection{Clinical Outcome}

\section{VT Recurrence}

During the median follow-up period of 1.4 years (IQR, 0.3-2.0), $22(34 \%)$ of the cases experienced VT recurrences (Table 4 and Figure 3). 


\section{Predictive factors of VT recurrence}

Table 5 shows the univariable and multivariable Cox regression analyses of VT recurrence. In the univariable analysis, an LVEF[?] 35\% (HR 0.20; 95\% CI 0.06- 0.51; P=0.01) and successful identification and ablation of all clinical VT isthmuses (Group A) (HR 0.22; 95\% CI 0.03- 0.74; $\mathrm{P}=0.01$ ) were associated with fewer VT recurrences (Figure 4). A Cox proportional model was used for the multivariate analysis, and variables with a $\mathrm{P}<0.10$ in the univariable analysis were included in the model. An LVEF[?]35\% (HR 0.21; 95\% CI 0.070.54; $\mathrm{P}<0.01$ ) and the successful identification and ablation of all clinical VT isthmused (Group A) (HR 0.21; $95 \%$ CI $0.03-0.72 ; \mathrm{P}=0.01$ ) were independent predictors of fewer VT recurrences in the multivariate analysis.

\section{VT burden reduction}

Figure 5 shows the VT episodes before and after the RF ablation. A significant VT burden reduction after the VT ablation was observed in the entire population. RF ablation was associated with a $91.1 \%$ reduction in VT episodes. Before and after the RF ablation, the median VT episodes were 3 (IQR, 2 - 9) and 0 (IQR, 0 - 1) $(\mathrm{P}<0.01)$, and the number of shock therapies was $2(\mathrm{IQR}, 1-5)$ and $0(\mathrm{IQR}, 0-0)(\mathrm{P}<0.01)$.

\section{Mortality}

Fourteen patients died during the follow-up. The cause of death was arrhythmogenic cardiac death (29\%), terminal heart failure (36\%), and non-cardiovascular death (36\%) (Table 4).

\section{Discussion}

\section{Main Findings}

We evaluated the VT recurrence in patients in whom VT non-inducibility could not be achieved at the end of the RF ablation and the factors attributed to the VT recurrence in ICM and NICM patients.

The main findings of our study were:

1. During the median follow-up period of 1.4 years (IQR, 0.3-2.0), $66 \%$ of the patients in whom VT noninducibility could not be achieved at the end of RF ablation had no recurrence.

2. Patients whose LVEF was more than or equal to $35 \%$, or in whom all clinical VT isthmuses could successfully be identify and ablated were independent predictors of fewer VT recurrences in the multivariate analysis.

3. Even in patients with VT recurrences, VT ablation could significantly reduce the VT burden.

Endpoint of the VT ablationIt is known that VT non-inducibility at the end of the VT ablation is less likely to be associate with a VT recurrence ${ }^{6,7}$ and achieving the combined endpoint of the abolition of the abnormal electrograms and VT non-inducibility further reduces the VT recurrence. ${ }^{8,9}$ In the present study, 20 patients in whom VT non-inducibility was achieved had a small VT recurrence rate of $15 \%$. However, we need to consider the risk of overtreatment at the same time; the enlargement of a scar area by RF ablation may lead to a more depressed cardiac function. There is not enough detailed reporting on the subsequent events in patients with residual VT inducibility at the end of the procedure. In our study, patients with an LVEF[?]35\% or successful identification and ablation of all clinical VT isthmuses were independent predictors of VT non-recurrence in patients in whom VT non-inducibility was not achieved. This may indicate that we should try to identify the isthmus of the clinical VT as much as possible, and that in this group of patients, inducible residual non-clinical VT is acceptable.

\section{LV dysfunction and VT recurrence}

In this study, the recurrence rate was lower in the patients with an LVEF[?]35\%, even with residual VT inducibility. In a sub-analysis of the AVID (Antiarrhythmics Versus Implantable Defibrillator) trial, the efficacy of ICD therapy was reported to depend on the degree of LV dysfunction in patients with secondary prevention of sudden cardiac death, and in patients with an LVEF $<35 \%$, ICD therapy had an advantage. 
On the other hand, in patients with an LVEF[?]35\%, the efficacy of the ICD therapy was equivalent to that of antiarrhythmic drugs. ${ }^{12}$ Also in a recent trial, Groeneveld et al. reported that in 42 patients with an LVEF $>35 \%$ and a hemodynamically not tolerated VT, only 6 patients (14.3\%) had VT recurrences, and all were hemodynamically tolerated. ${ }^{13}$ That suggests that the classification by the degree of LV dysfunction may make sense, and may help determine how aggressively induced VT should be treated.

Patients with an $\mathrm{EF}<35 \%$ are considered to have more myocardial dysfunction and are more likely to have multiple arrhythmia substrates. Although a more aggressive induction and ablation may be necessary to reduce the VT recurrence rate in them, the dilemma is that patients with such a reduced cardiac function may be more susceptible to hemodynamic compromise and invasive procedures with a prolonged operative time. In the present study, the VT burden was significantly reduced even in the group with an EF $<35 \%$, suggesting that a balanced protocol that ensures safety is desirable.

\section{Importance of a successful ablation of all clinical VT isthmuses}

Previous randomized multicenter trials have shown that substate ablation reduces any VT recurrences during the follow-up as compared to targeting only a clinical and stable VT, making substate ablation a basic strategy for VT ablation. ${ }^{14-16}$ In the present study, the identification and RF ablation of the clinical VT isthmus in addition to a substate ablation was shown to be associated with fewer VT recurrences. Hadjis et al. reported that the identification of the VT isthmus in addition to a substate ablation significantly reduced the recurrence rate (HR 0.21, 95\% CI: 0.07-0.63, P < 0.01). ${ }^{17}$ Cano et al. also reported that a baseline inducibility of greater than 1 VT morphology was an independent predictor of VT recurrence (HR 12.05, $95 \%$ CI: $1.60-90.79, \mathrm{P}=0.02)$ and complete activation mapping was associated with a reduction in the VT recurrence. ${ }^{18}$

In this study, patients in whom VT non-inducibility could not be achieved at the end of the RF ablation were included, so in many cases, VT was induced with or without hemodynamic stability at the end of the RF ablation. Nevertheless, a successful ablation of all clinical VT isthmuses was correlated with non-recurrence, suggesting that the identification of the VT isthmus is important in addition to the basic strategy of substate ablation.

\section{VT burden reduction}

The term "recurrence" has been used to evaluate the outcome of the ablation therapy, however, recently the concept of a VT burden reduction has been proposed. ${ }^{19,} 20$ The VT burden can be more valuable in terms of clinical benefit than recurrence as a dichotomous event. In the present study, the VT burden was reduced by $91.1 \%$ even in patients in whom VT non-inducibility could not be achieved at the end of RF ablation, and VT ablation significantly reduced the number of VT episodes and shock therapies. Although the design of the current study did not allow us to examine the prevention of mortality, a reduction in the ICD shock therapies may improve the patient survival. ${ }^{21}$

\section{Clinical Implications}

It is known that the achievement of VT non-inducibility at the end of the VT ablation in patients with SHD leads to a lower recurrence. So VT non-inducibility has been used as an endpoint in many cases. However, in some cases, the ablation may be excessive or the risk of an induction test may be high. In this study, we included patients in whom VT non-inducibility could not achieved at the end of the VT ablation. We found that the recurrence rate was lower in patients with an EF[?]35\% or in patients who could successfully identify and undergo ablation of all clinical VT isthmuses. In these patients, even if nonclinical VT inducibility remains, it may not affect the subsequent prognosis. In addition, the VT burden was reduced after the VT ablation, and the shock therapy was also significantly reduced before and after the ablation. That suggested that it may be necessary to examine the validity of the VT non-inducibility in each patient.

\section{Study Limitations}


This study was a single-center retrospective observational analysis. Therefore, the number of study patients was relatively small. And this study included the patients with ICM and NICM, and the NICM consisted of a non-uniform etiology. The response to the RF ablation and clinical course may differ for each cardiomyopathy. Further, the methods used for antiarrhythmic drugs were dependent on each physician. However, because of the limited number of operators, our strategy to treat patients with VTs has been consistent and our results can be applied to clinical practice.

\section{Conclusion}

Even if VT non-inducibility could not be achieved at the end of the RF ablation, $66 \%$ of the patients had no VT recurrences. Among them, in the patients whose LVEF was more than or equal to $35 \%$ or in whom all clinical VT isthmuses could be identified and ablated, that successful identification and ablation of those might prevent VT recurrences. In addition, even if the VT recurred, the VT burden decreased after the VT ablation. The validity of VT non-inducibility for any VT should be evaluated by each patient's background and the results of the procedure.

\section{Acknowledgments}

The authors would like to thank Mr. John Martin for his linguistic assistance.

\section{References}

1. Kuck K-H, Schaumann A, Eckardt L, Willems S, Ventura R, Delacretaz E, Pitschner H-F, Kautzner J, Schumacher B and Hansen PS. Catheter ablation of stable ventricular tachycardia before defibrillator implantation in patients with coronary heart disease (VTACH): a multicentre randomised controlled trial. The Lancet . 2010;375:31-40.

2. Marchlinski FE, Haffajee CI, Beshai JF, Dickfeld T-ML, Gonzalez MD, Hsia HH, Schuger CD, Beckman KJ, Bogun FM and Pollak SJ. Long-term success of irrigated radiofrequency catheter ablation of sustained ventricular tachycardia: post-approval THERMOCOOL VT trial.Journal of the American College of Cardiology . 2016;67:674-683.

3. Reddy VY, Reynolds MR, Neuzil P, Richardson AW, Taborsky M, Jongnarangsin K, Kralovec S, Sediva L, Ruskin JN and Josephson ME. Prophylactic catheter ablation for the prevention of defibrillator therapy. New England Journal of Medicine . 2007;357:2657-2665.

4. Gonska B-D, Cao K, Schaumann A, Dorszewski A, von zur Muhlen F and Kreuzer H. Catheter ablation of ventricular tachycardia in 136 patients with coronary artery disease: results and long-term follow-up.Journal of the American College of Cardiology . 1994;24:1506-1514.

5. Jais P, Maury P, Khairy P, Sacher F, Nault I, Komatsu Y, Hocini M, Forclaz A, Jadidi AS and Weerasooryia R. Elimination of local abnormal ventricular activities: a new end point for substrate modification in patients with scar-related ventricular tachycardia. Circulation . 2012;125:2184-2196.

6. Dinov B, Arya A, Schratter A, Schirripa V, Fiedler L, Sommer P, Bollmann A, Rolf S, Piorkowski C and Hindricks G. Catheter ablation of ventricular tachycardia and mortality in patients with nonischemic dilated cardiomyopathy: can noninducibility after ablation be a predictor for reduced mortality? Circulation: Arrhythmia and Electrophysiology . 2015;8:598-605.

7. Ghanbari H, Baser K, Yokokawa M, Stevenson W, Della Bella P, Vergara P, Deneke T, Kuck K-H, Kottkamp $\mathrm{H}$ and Fei S. Noninducibility in postinfarction ventricular tachycardia as an end point for ventricular tachycardia ablation and its effects on outcomes: a meta-analysis. Circulation: Arrhythmia and Electrophysiology . 2014;7:677-683.

8. Okubo K, Gigli L, Trevisi N, Foppoli L, Radinovic A, Bisceglia C, Frontera A, D'Angelo G, Cireddu M and Paglino G. Long-Term Outcome After Ventricular Tachycardia Ablation in Nonischemic Cardiomyopathy: Late Potential Abolition and VT Noninducibility. Circulation: Arrhythmia and Electrophysiology . 2020;13:e008307. 
9. Silberbauer J, Oloriz T, Maccabelli G, Tsiachris D, Baratto F, Vergara P, Mizuno H, Bisceglia C, Marzi A and Sora N. Noninducibility and late potential abolition: a novel combined prognostic procedural end point for catheter ablation of postinfarction ventricular tachycardia.Circulation: Arrhythmia and Electrophysiology . 2014;7:424-435.

10. Fukuzawa K, Nagamatsu Y, Mori S, Kiuchi K, Takami M, Izawa Y, Konishi H, Ichibori H, Imada H and Hyogo K. Percutaneous pericardiocentesis with the anterior approach: demonstration of the precise course with computed tomography. JACC: Clinical Electrophysiology . 2019;5:730-741.

11. Tung R, Mathuria N, Michowitz Y, Yu R, Buch E, Bradfield J, Mandapati R, Wiener I, Boyle N and Shivkumar K. Functional pace-mapping responses for identification of targets for catheter ablation of scar-mediated ventricular tachycardia. Circulation: Arrhythmia and Electrophysiology . 2012;5:264-272.

12. Domanski MJ, Sakseena S, Epstein AE, Hallstrom AP, Brodsky MA, Kim S, Lancaster S, Schron E and Investigators A. Relative effectiveness of the implantable cardioverter-defibrillator and antiarrhythmic drugs in patients with varying degrees of left ventricular dysfunction who have survived malignant ventricular arrhythmias. Journal of the American College of Cardiology . 1999;34:1090-1095.

13. Groeneveld SA, Blom LJ, van der Heijden JF, Loh P and Hassink RJ. Follow-up after hemodynamically not tolerated ventricular tachycardia in patients with midrange reduced to normal ejection fraction: A retrospective single-centre case series. European Journal of Clinical Investigation . 2021;51:e13359.

14. Briceno DF, Romero J, Villablanca PA, Londono A, Diaz JC, Maraj I, Batul SA, Madan N, Patel J and Jagannath A. Long-term outcomes of different ablation strategies for ventricular tachycardia in patients with structural heart disease: systematic review and meta-analysis. Ep Europace . 2018;20:104-115.

15. Di Biase L, Burkhardt JD, Lakkireddy D, Carbucicchio C, Mohanty S, Mohanty P, Trivedi C, Santangeli P, Bai R and Forleo G. Ablation of stable VTs versus substrate ablation in ischemic cardiomyopathy: the VISTA randomized multicenter trial. Journal of the American College of Cardiology . 2015;66:2872-2882.

16. Kumar S, Baldinger SH, Romero J, Fujii A, Mahida SN, Tedrow UB and Stevenson WG. Substratebased ablation versus ablation guided by activation and entrainment mapping for ventricular tachycardia: a systematic review and meta-analysis. Journal of cardiovascular electrophysiology . 2016;27:1437-1447.

17. Hadjis A, Frontera A, Limite LR, Bisceglia C, Bognoni L, Foppoli L, Lipartiti F, Paglino G, Radinovic A and Tsitsinakis G. Complete electroanatomic imaging of the diastolic pathway is associated with improved freedom from ventricular tachycardia recurrence.Circulation: Arrhythmia and Electrophysiology . 2020;13:e008651.

18. Cano O, Perez-Rosello V, Ayala HD, Izquierdo M, Osca J, Sancho-Tello MJ and Martinez-Dolz L. Influence of baseline inducibility and activation mapping on ablation outcomes in patients with structural heart disease and ventricular tachycardia. Journal of Cardiovascular Electrophysiology . 2021;32:1328-1336.

19. Quinto L, Sanchez-Somonte P, Alarcon F, Garre P, Castillo A, San Antonio R, Borras R, Guasch E, Arbelo E and Tolosana JM. Ventricular tachycardia burden reduction after substrate ablation: predictors of recurrence. Heart Rhythm . 2021;18:896-904.

20. Wolf M, Sacher F, Cochet H, Kitamura T, Takigawa M, Yamashita S, Vlachos K, Cheniti G, Frontera A and Martin R. Long-term outcome of substrate modification in ablation of post-myocardial infarction ventricular tachycardia. Circulation: Arrhythmia and Electrophysiology . 2018;11:e005635.

21. Poole JE, Johnson GW, Hellkamp AS, Anderson J, Callans DJ, Raitt MH, Reddy RK, Marchlinski FE, Yee R and Guarnieri T. Prognostic importance of defibrillator shocks in patients with heart failure. New England Journal of Medicine . 2008;359:1009-1017.

\section{Hosted file}


scar-related VT_Figure_nakasone.docx available at https://authorea.com/users/371626/articles/ 548238-predictors-of-vt-recurrence-in-patients-with-vt-inducibility-at-the-end-ofradiofrequency-ablation-should-we-use-vt-non-inducibility-as-a-routine-endpoint

\section{Hosted file}

scar-related VT_Table_nakasone.docx available at https://authorea.com/users/371626/articles/ 548238-predictors-of-vt-recurrence-in-patients-with-vt-inducibility-at-the-end-ofradiofrequency-ablation-should-we-use-vt-non-inducibility-as-a-routine-endpoint 